\title{
Impact of allergic rhinitis on asthma: effects on bronchodilation testing
}

Giorgio Ciprandi, MD*; Ignazio Cirillo, MD†; Angela Pistorio, MD‡; Stefania La Grutta, MD\$; and Mariangela Tosca, MDII

Background: A remarkable relationship exists between the upper and lower airways. Bronchial obstruction is a paramount feature of asthma, and its reversibility is considered a main step in asthma diagnosis.

Objective: To investigate the degree of bronchodilation and possible risk factors related to it in patients with moderate-severe persistent allergic rhinitis alone.

Methods: A total of 375 patients with moderate-severe persistent allergic rhinitis and 115 controls were prospectively and consecutively evaluated by means of clinical examination, skin prick testing, spirometry, and bronchodilation testing.

Results: Patients with rhinitis showed a significant increase in forced expiratory volume in 1 second (FEV) after bronchodilation testing compared with basal values and levels in controls $(P<.001)$. Two-thirds of the rhinitic patients had reversibility ( $\geq 12 \%$ basal levels). Patients with reversibility had lower $\mathrm{FEV}_{1}$ levels, longer rhinitis duration, and mite and tree allergies.

Conclusions: This study highlights the close link between the upper and lower airways and the relevance of performing bronchodilation testing in patients with moderate-severe persistent allergic rhinitis.

Ann Allergy Asthma Immunol. 2008;101:42-46.

\section{INTRODUCTION}

Allergic rhinitis is characterized by typical symptoms, including nasal itching, sneezing, rhinorrhea, and obstruction, induced by an IgE-mediated inflammatory response of the nose to allergen exposure. Indeed, inflammation may be considered a condicio sine qua non of allergic rhinitis. ${ }^{1}$ Infiltration by inflammatory cells, including $\mathrm{T}$ cells, mast cells, and eosinophils, characterizes allergic inflammation. ${ }^{2}$ The cytokine pattern is typically characterized by a $\mathrm{T}_{\mathrm{H}} 2$ polarization. ${ }^{3}$ The $\mathrm{T}_{\mathrm{H}}$ 2-derived cytokines account for recruiting and activating eosinophils in the airways: actually, eosinophil infiltration may be considered a reliable marker of allergic inflammation. ${ }^{4}$

On the other hand, asthma is defined as chronic inflammation of the lower airways. ${ }^{5}$ The allergic airway inflammation may, therefore, induce airflow limitation at the nasal and bronchial levels. ${ }^{6} \mathrm{~A}$ close link between allergic rhinitis and asthma has been widely reported. ${ }^{7,8}$ Furthermore, allergic rhinitis has been demonstrated to be a strong risk factor for the onset of asthma in adults. ${ }^{9}$

From a pathophysiologic point of view, asthma is characterized by airflow obstruction. ${ }^{10,11}$ Bronchial airflow may be easily evaluated by means of spirometry. Several spirometric variables may be considered, but the gold standard for detecting asthma is forced expiratory volume in 1 second

Affiliations: * Department of Internal Medicine, Azienda Ospedaliera Universitaria San Martino, Genoa, Italy; $\dagger$ Navy Medical Service, La Spezia, Italy; $\ddagger$ Epidemiology and Statistics Unit, IRCCS G. Gaslini, Genoa, Italy; $\S$ Environment and Health Unit, ARPA Sicilia, and IBIM CNR, Palermo, Italy; II Allergy Center, IRCCS G. Gaslini, Genoa, Italy.

Disclosures: Authors have nothing to disclose.

Received for publication January 21, 2008; Received in revised form March 5, 2008; Accepted for publication March 23, 2008.
$\left(\mathrm{FEV}_{1}\right)$ according to Global Initiative for Asthma guidelines. ${ }^{5}$ In addition, reversibility of airflow obstruction is considered a characteristic of asthma. This reversibility may be spontaneous or induced by drugs such as bronchodilators. To evaluate reversibility, the bronchodilation test is commonly performed in a clinical setting.

Because allergic rhinitis may frequently be associated with or precede asthma, the World Health Organization document Allergic Rhinitis and Its Impact on Asthma ${ }^{6}$ clearly underlines the role of allergic rhinitis as a risk factor for asthma and suggests considering bronchial involvement in patients with allergic rhinitis. Concerning this issue, it has been demonstrated that $\mathrm{FEV}_{1}$ may be impaired in approximately $5 \%$ of patients with allergic rhinitis who perceive nasal symptoms alone. ${ }^{12}$ Furthermore, slight spirometric impairment, such as reduced forced expiratory flow between $25 \%$ and $75 \%$ values, may frequently be found in rhinitic patients, and it might be a reliable marker of early bronchial involvement. ${ }^{13}$ Therefore, allergic rhinitis may be considered the first step in a progression of respiratory allergy toward asthma. Furthermore, the Allergic Rhinitis and Its Impact on Asthma document revised the classification of allergic rhinitis, defining 2 types: intermittent and persistent. The new classification does not consider the type of allergen but the duration (days per week and consecutive weeks) and the severity of symptoms (mild or moderate-severe).

Nevertheless, the possible presence of spirometric abnormalities in patients with allergic rhinitis has been well documented; however, no studies, to our knowledge, have been conducted in patients with moderate-severe persistent allergic rhinitis about the degree of reversibility to bronchodilation testing. Therefore, a large group of patients with moderatesevere persistent allergic rhinitis alone were evaluated to 
Table 1. Characteristics of the 375 Study Patients

\begin{tabular}{lc}
\hline \multicolumn{1}{c}{ Characteristic } & Value \\
\hline Female sex, No. (\%) & $77(20.5)$ \\
Age, median (range), y & $24(18-48)$ \\
Rhinitis duration, median (range), y & $3(1-12)$ \\
Pretest FEV ${ }_{1}$, median (range), \% of predicted & $90(80-109)$ \\
Pretest FVC, median (range), \% of predicted & $102(77-116)$ \\
Pretest $_{\text {FEF }}{ }_{25-75}$, median (range), \% of predicted & $72(56-79)$ \\
\hline
\end{tabular}

Abbreviations: $\mathrm{FEF}_{25-75}$, forced expiratory flow between $25 \%$ and $75 \%$; $\mathrm{FEV}_{1}$, forced expiratory volume in 1 second; FVC, forced vital capacity.

investigate the degree of bronchodilation and possible risk factors related to it.

\section{MATERIALS AND METHODS}

\section{Study Design and Participants}

The study included 375 patients with moderate-severe persistent allergic rhinitis who were prospectively and consecutively evaluated using clinical examination, skin prick testing, spirometry, and bronchodilation testing. A total of 115 individuals were enrolled as controls. Patient characteristics, including sex, age, and duration of rhinitis, are reported in Table 1. All the patients were Navy sailors who had been referred to a Navy hospital for a mandatory periodic visit for maintaining the qualification; therefore, this study is exempt from institutional review board review. Informed consent was obtained from each participant.

A detailed clinical history was taken, and a complete physical examination was performed. Patients were included in the study on the basis of a clinical history of persistent allergic rhinitis and the presence of moderate-severe nasal symptoms according to validated criteria. ${ }^{6}$ The exclusion criteria were as follows: any history of asthma or the presence of asthma symptoms, including cough, wheezing, dyspnea, and shortness of breathing; acute or chronic upper respiratory tract infections; anatomical nasal disorders (nasal polyps, septum deviation); previous or current smoking (screened by expired carbon dioxide evaluation); previous or current specific immunotherapy; and use of nasal or oral corticosteroids, nasal or oral vasoconstrictors, antileukotrienes, and antihistamines during the previous 4 weeks (if they assumed pharmacologic treatment, they were asked to return after stopping the medication use for 4 weeks). All the patients were treated on demand only with drugs alone. The diagnosis of persistent allergic rhinitis was made on the basis of a history of nasal symptoms and positive skin prick test results according to validated criteria. $^{6}$

\section{Testing}

Skin prick testing was performed as stated by the European Academy of Allergy and Clinical Immunology. ${ }^{14}$ The panel consisted of house dust mites (Dermatophagoides farinae and Dermatophagoides pteronyssinus), cat, dog, grass mix, Compositae mix, Parietaria officinalis, birch, hazel, olive tree,
Alternaria tenuis, Cladosporium, and Aspergilli mix (Stallergenes, Milan, Italy). Spirometry was performed using a computer-assisted spirometer (Pulmolab 435-Spiro 235; Morgan Medical, Rainham, England) and according to international guidelines. ${ }^{5,10,11}$ Briefly, 3 blows (every 5 minutes) were performed, and the best result was considered. Test of bronchodilation was performed according to international guidelines and using a salbutamol metered dose of $400 \mu \mathrm{g}$. Reversibility was considered if an increase of at least $12 \%$ in $\mathrm{FEV}_{1}$ from baseline was achieved, according to international guidelines. ${ }^{10,11}$

\section{Statistical Analysis}

Descriptive statistical analyses were performed, and quantitative variables are reported as mean (SD) or as median (range) in the case of skewed distribution. Qualitative data are reported as frequency (percentage). Qualitative data were compared among various groups of patients using the $\chi^{2}$ test or the Fisher exact test in the case of expected frequencies less than 5. Quantitative normally distributed data were compared between 2 groups of patients using the $t$ test; when the homoscedasticity assumption was not fulfilled, the MannWhitney test was used as a nonparametric counterpart (eg, age of the patients). Quantitative variables were compared among the 3 groups (such as patients with persistent allergic rhinitis and reversibility, patients with persistent allergic rhinitis without reversibility, and control subjects) by means of analysis of variance or the nonparametric Kruskal-Wallis test; post hoc comparisons were performed using the Scheffé test or the nonparametric Dunn test.

Quantitative variables (eg, $\mathrm{FEV}_{1} \%$ of predicted) for paired data (pretest and posttest values) were compared using the Wilcoxon test. All tests were 2 sided, and $P<.05$ was considered statistically significant. A statistical software program (Statistica release 6; StatSoft Corp, Tulsa, Oklahoma) was used for all the analyses.

\section{RESULTS}

Three hundred seventy-five patients (298 men and 77 women) and 115 control subjects (102 men and 13 women) were included in the study. The mean (SD) age of patients was 23.9 (3.9) years and of controls was 22.6 (2.4) years (Mann-Whitney test, $P=.31$ ). Two hundred fifty-four patients had a diagnosis of allergic rhinitis, and 121 had a diagnosis of allergic rhinoconjunctivitis. All of the included individuals have clinically relevant sensitization to at least 1 allergen to which they were exposed at the time of testing.

Patients had a median rhinitis duration of 3 years, and none had $\mathrm{FEV}_{1}$ values less than $80 \%$ of predicted (Table 1). Thus, patients were studied on the basis of their response to bronchodilation testing. Figure 1A shows pretest and posttest $\mathrm{FEV}_{1}$ values in patients and controls. Mean pretest $\mathrm{FEV}_{1}$ values are approximately $90 \%$ of predicted (mean [SD], $89.8 \%$ [6.1\%] of predicted; median, $90.0 \%$ of predicted) and reach a mean (SD) posttest value of $100.8 \%$ (5.2\%) of predicted (median, $102.0 \%$ of predicted). This increase is statis- 

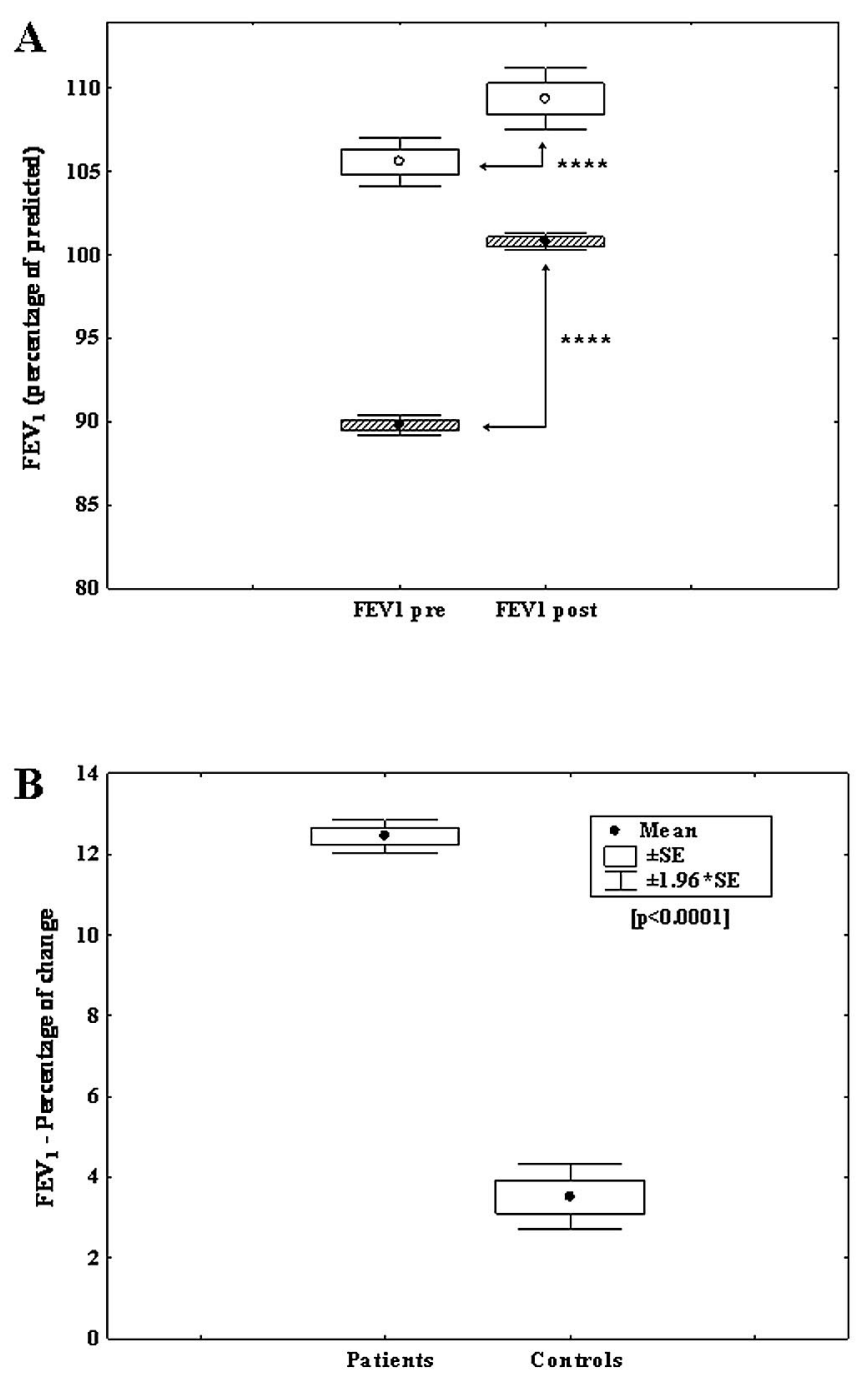

Figure 1. A, Pretest and posttest forced expiratory volume in 1 second $\left(\mathrm{FEV}_{1}\right)$ values in patients (striped boxes) and controls (white boxes). $* * * * P<.001$. B Percentage change in $\mathrm{FEV}_{1}$ values is significantly higher in patients vs controls. Error bars represent SD.

tically significant (Wilcoxon test, $P<.001$ ). In addition, pretest and posttest $\mathrm{FEV}_{1}$ values are higher in controls than in patients; pretest and posttest values in controls also show a significant increase (pretest $\mathrm{FEV}_{1}$ values: mean [SD], 105.6\% [8.2\%] of predicted; median, $105 \%$ of predicted; and posttest $\mathrm{FEV}_{1}$ values: mean [SD], $109.4 \%$ [10.1\%] of predicted; median, $112 \%$ of predicted) (Wilcoxon test, $P<.001$ ) (Fig 1A). Furthermore, posttest $\mathrm{FEV}_{1}$ values remain significantly lower in patients than in controls (Mann-Whitney test, $P<.001$ ).

The percentage change $(\Delta)$ in $\mathrm{FEV}_{1}$ values (with respect to pretest values) is significantly higher in patients vs controls (Mann-Whitney test, $P<.001$ ) (Fig 1B). Of the 375 patients, $251(66.9 \%)$ showed a percentage increase in $\mathrm{FEV}_{1}$ of $12 \%$ or greater and, therefore, had reversibility, whereas $124(33.1 \%)$ showed a percentage increase in $\mathrm{FEV}_{1}$ of less than $12 \%$. None of the controls was "reversible"; in fact, all the percentage increases in $\mathrm{FEV}_{1}$ in the control group were less than $12 \%$.

Mean $\mathrm{FEV}_{1}$ values at baseline in the 3 participant groups are shown in Figure 2A. Patients with a $\Delta \mathrm{FEV}_{1} \%$ of predicted of $12 \%$ or greater have lower levels of pretest $\mathrm{FEV}_{1}$ vs patients with a $\Delta \mathrm{FEV}_{1}$ of less than $12 \%$ or controls (Dunn test, $P<.01$ for both). As shown in Figure 2B, rhinitis duration is significantly higher in patients with a $\Delta \mathrm{FEV}_{1}$ of $12 \%$ or greater compared with patients with a $\Delta \mathrm{FEV}_{1}$ of less than $12 \%$.

Allergen sensitization in the 2 groups of patients $\left(\Delta \mathrm{FEV}_{1}\right.$ $\geq 12 \%$ vs $\Delta \mathrm{FEV}_{1}<12 \%$ ) is given in Table 2 . Patients with
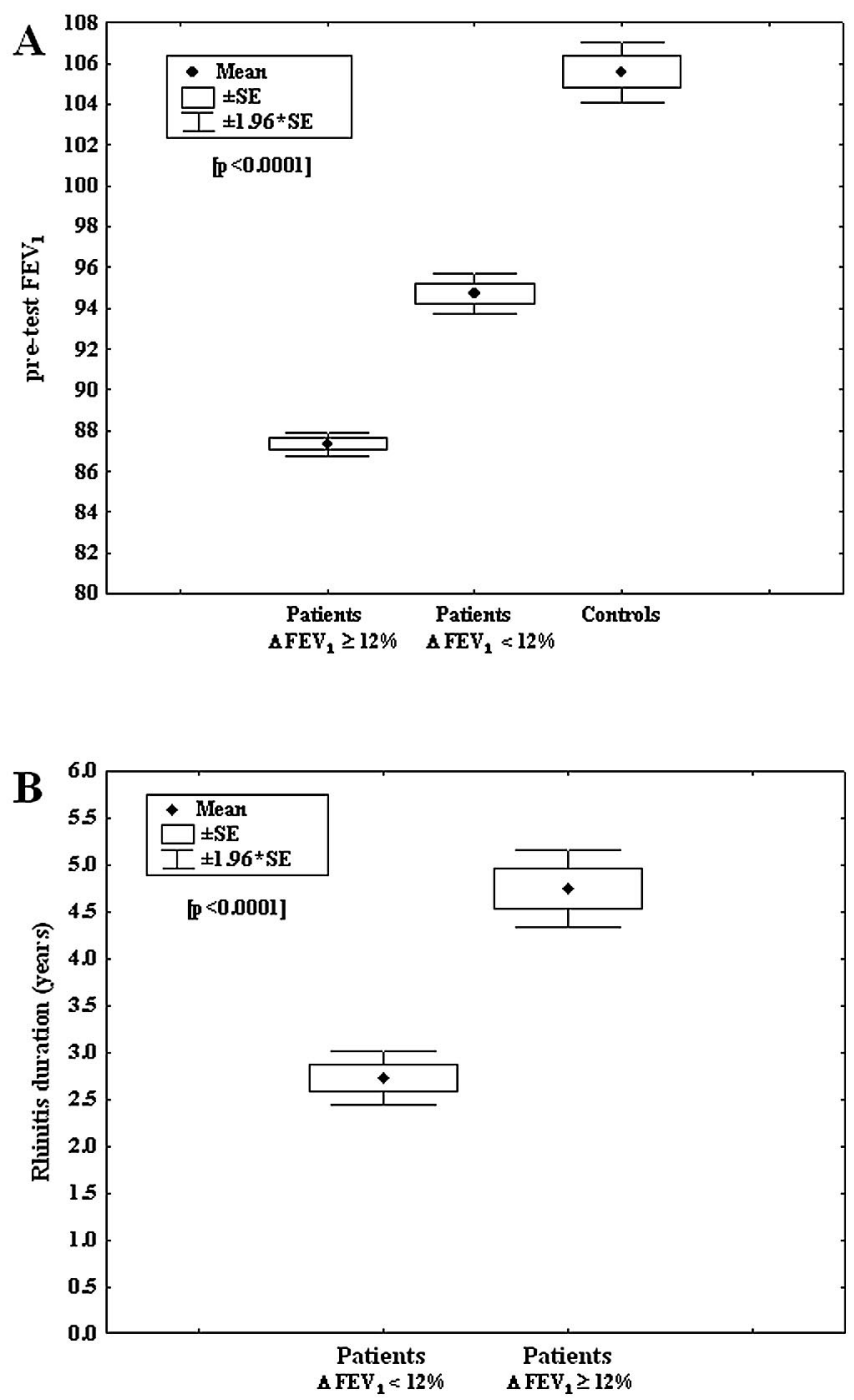

Figure 2. A, Pretest forced expiratory volume in 1 second $\left(\mathrm{FEV}_{1}\right)$ values in the 3 groups: patients with a percentage change $(\Delta)$ in $\mathrm{FEV}_{1} \%$ of predicted of $12 \%$ or greater, patients with a $\Delta \mathrm{FEV}_{1}$ of less than $12 \%$, and controls (all with a $\Delta \mathrm{FEV}_{1}$ of $<12 \%$ ). B, Rhinitis duration is significantly higher in patients with a $\Delta \mathrm{FEV}_{1} \%$ of predicted of $12 \%$ or greater. 
Table 2. Allergen Sensitization in the 375 Study Patients Divided in 2 Groups According to Reversibility

\begin{tabular}{|c|c|c|c|}
\hline \multirow{2}{*}{ Sensitization to } & \multicolumn{2}{|c|}{ Percentage change in $\mathrm{FEV}_{1} \%$ of predicted } & \multirow{2}{*}{$P$ value ( $\chi^{2}$ test) } \\
\hline & $\geq 12 \%(n=251)$ & $<12 \%(n=124)$ & \\
\hline House dust mites, No. (\%) & $183(72.9)$ & $73(58.9)$ & .006 \\
\hline Trees, No. (\%) & $146(58.2)$ & $46(37.1)$ & $<.001$ \\
\hline Parietaria, №. (\%) & $160(63.7)$ & $70(56.5)$ & .17 \\
\hline Grass, No. (\%) & 35 (13.9) & $15(12.1)$ & .62 \\
\hline Molds, No. (\%) & $34(13.5)$ & $34(27.4)$ & .001 \\
\hline Dog or cat, No. (\%) & $17(6.8)$ & $9(7.3)$ & .86 \\
\hline Compositae, No. (\%) & $6(2.4)$ & $7(5.6)$ & .13 \\
\hline
\end{tabular}

$\Delta \mathrm{FEV}_{1}$ of $12 \%$ or greater are more frequently positive to house dust mites and trees and are less frequently positive to molds.

\section{DISCUSSION}

Allergic rhinitis and asthma may be considered as a single syndrome involving 2 parts of the respiratory tract, as documented by 2 elegant experimental studies. ${ }^{15,16}$ Patients with allergic rhinitis may frequently present with asthma symptoms or spirometric impairment. Indeed, impaired $\mathrm{FEV}_{1}$ values may be detected in some patients with allergic rhinitis who also perceive nasal symptoms alone..$^{12}$ Thus, this finding underlines the link between the upper and lower airways and underlines the concept that allergic rhinitis usually precedes overt asthma.

Asthma is characterized by airflow obstruction that is typically reversible, spontaneously or pharmacologically. The demonstration of bronchial reversibility is a main step in the diagnosis of asthma and is performed by bronchodilation testing. Bronchodilation testing should be considered as an integral part of spirometry because it is easy to perform and may provide interesting suggestions also in patients without overt bronchial airflow obstruction.

The present study investigated the response to the bronchodilation test in a large cohort of patients with moderatesevere persistent allergic rhinitis alone, ie, perceiving only nasal symptoms. Several interesting findings have been observed in this study. First, it provides evidence that a large percentage (approximately two-thirds) of patients with moderate-severe persistent allergic rhinitis show reversibility to bronchodilation testing (ie, an increase greater than $12 \%$ in basal $\mathrm{FEV}_{1}$ values). Note that all the patients had normal basal $\mathrm{FEV}_{1}$ values (ie, $80 \%$ of predicted or greater). Furthermore, bronchodilation has been observed also in patients without reversibility (ie, less than a $12 \%$ increase in basal $\mathrm{FEV}_{1}$ values), and the increase in $\mathrm{FEV}_{1}$ values is significantly higher than in controls. Therefore, it is conceivable to formulate the hypothesis that the response to bronchodilation testing might be considered as further proof of bronchial impairment in allergic rhinitis.

Second, the response degree to bronchodilation testing depends on basal $\mathrm{FEV}_{1}$ values. Indeed, patients with reversibility had significantly lower $\mathrm{FEV}_{1}$ values than did patients without reversibility. This issue underlines the concept that reversibility to bronchodilation testing is associated with early bronchial airflow limitation, although in the presence of borderline $\mathrm{FEV}_{1}$ values.

Third, reversibility is associated with longer duration of rhinitis. This finding confirms that of a previous study ${ }^{17}$ providing evidence that spirometric impairments are more frequent in patients with long-lasting rhinitis. The duration of allergic rhinitis constitutes an important factor that promotes the development of bronchial involvement. The possible explanation of this phenomenon might depend on the allergic inflammation that persists across time and tends to progress from the nose into the bronchi. ${ }^{6}$

Fourth, the reversibility is associated with specific sensitizations (ie, to house dust mites and trees). This fact confirms the previous outcome: mite allergy is characterized by the well-known concept of minimal persistent inflammation. ${ }^{18}$ The continuous exposure to causal allergen induces persistence of inflammation that may favor complications. In addition, it has been evidenced that birch allergy is also characterized by this phenomenon. ${ }^{19}$ Therefore, as in asthmatic patients also in rhinitic patients the response to bronchodilation testing seems to depend on airway inflammation. The implication of this study is that in addition to having upper airway inflammation, these allergic rhinitic patients also have lower airway inflammation, which may be contributing to their lower $\mathrm{FEV}_{1}$ values and significant reversibility on spirometry.

In conclusion, this study provides evidence that patients with moderate-severe persistent allergic rhinitis may frequently show reversibility to bronchodilation testing. This event may account for early bronchial involvement as patients were perceiving nasal symptoms alone. The reversibility is also associated with lower basal $\mathrm{FEV}_{1}$ values, longer duration of rhinitis, and sensitization to mites or trees. Thus, it could be useful to perform bronchodilation testing in patients with these characteristics.

\section{ACKNOWLEDGMENTS}

We thank Professor Giovanni Viegi for his helpful suggestions. 


\section{REFERENCES}

1. Christodoulopoulos P, Cameron L, Durham S, Hamid Q. Molecular pathology of allergic disease, II: upper airway disease. J Allergy Clin Immunol. 2000;105:211-223.

2. Foresi A, Leone C, Pelucchi A, et al. Eosinophils, mast cells, and basophils in induced sputum from patients with seasonal allergic rhinitis and perennial asthma: relationship to methacholine responsiveness. $J$ Allergy Clin Immunol. 1997;100:58-64.

3. Hamid QA, Minshall EM. Molecular pathology of allergic disease, I: lower airway disease. J Allergy Clin Immunol. 2000;105:20-36.

4. Ciprandi G, Vizzaccaro A, Cirillo I, Tosca MA, Massolo A, Passalacqua G. Nasal eosinophils display the best correlation with symptoms, pulmonary function and inflammation in allergic rhinitis. Int Arch Allergy Immunol. 2005;136:266-272.

5. Global Initiative for Asthma. Pocket Guide for Asthma Management and Prevention. Bethesda, MD: National Heart, Lung, and Blood Institute, National Institutes of Health; 1997. NIH publication 96-3659B.

6. Bousquet J, Van Cauwenberge P, Khaltaev N. Allergic rhinitis and its impact on asthma. J Allergy Clin Immunol. 2001;108(suppl): S147-S334.

7. Bugiani M, Carosso A, Migliore E, et al. Allergic rhinitis and asthma comorbidity in a survey of young adults in Italy. Allergy. 2005;60: 165-170.

8. Asher I. ISAAC International Study of Asthma and Allergies in Childhood. Pediatr Pulmonol. 2007;42:100.

9. Plaschke PP, Janson C, Norrman E. Onset and remission of allergic rhinitis and asthma and the relationship with atopic sensitization and smoking. Am J Respir Crit Care Med. 2000;162:920-924.

10. Pellegrino R, Viegi G, Brusasco V, et al. Interpretative strategies for lung function tests. Eur Respir J. 2005;26:948-968.

11. Miller MR, Hankinson J, Brusasco V, et al. Standardisation of spirometry. Eur Respir J. 2005;26:319-338.

12. Ciprandi G, Cirillo I, Vizzaccaro A, et al. Seasonal and perennial allergic rhinitis: is this classification adherent to real life? a population based study. Allergy. 2005;60:882-887.

13. Ciprandi G, Cirillo I, Klersy C, et al. Role of $\mathrm{FEF}_{25-75}$ as an early marker of bronchial impairment in patients with seasonal allergic rhinitis. Am J Rhinol. 2006;20:641-647.

14. Sub-Committee on Skin Tests of the European Academy of Allergology and Clinical Immunology. Skin tests used in type I allergy testing position paper. Allergy. 1989;44(suppl 10):1-59.

15. Braunstahl GJ, Kleinjan A, Overbeek SE, Prins JB, Hoogsteden HC, Fokkens WJ. Segmental bronchial provocation induces nasal inflammation in allergic rhinitis patients. Am J Respir Crit Care Med. 2000;161: 2051-2057.

16. Braunstahl GJ, Overbeek SE, Fokkens WJ, et al. Segmental bronchoprovocation in allergic rhinitis patients affects mast cell and basophil numbers in nasal and bronchial mucosa. Am J Respir Crit Care Med. 2001; $164: 858-865$.

17. Ciprandi G, Cirillo I, Pistorio A. Impact of allergic rhinitis on asthma effects on spirometric parameters. Allergy. 2008;63:255-260.

18. Ciprandi G, Buscaglia S, Pesce GP, et al. Minimal persistent inflammation is present at mucosal level in asymptomatic rhinitic patients with allergy due to mites. J Allergy Clin Immunol. 1995;96:971-979.

19. Ricca V, Landi M, Ferrero P, Bairo A, Tazzer C, Ciprandi G. Minimal persistent inflammation is present also in patients with seasonal allergic rhinitis. J Allergy Clin Immunol. 2000;105:54-57.

Requests for reprints should be addressed to:

Giorgio Ciprandi, $M D$

Semeiotica e Metodologia Medica I

Padiglione 3

A.O.U. San Martino

Largo R. Benzi 10

16132 Genoa, Italy

E-mail: gio.cip@libero.it 